\title{
REVIEW ARTICLE OPEN Therapeutic host-directed strategies to improve outcome in tuberculosis
}

\author{
C. Young ${ }^{1}$, G. Walzl ${ }^{1}$ and N. Du Plessis ${ }^{1}$
}

\begin{abstract}
Bacille Calmette-Guérin (BCG) is the only licenced tuberculosis (TB) vaccine, but has limited efficacy against pulmonary TB disease development and modest protection against extrapulmonary TB. Preventative antibiotic treatment for Mycobacterium tuberculosis $(M t b)$ infections in high-prevalence settings is unfeasible due to unclear treatment durability, drug toxicity, logistical constraints related to directly observed treatment strategy (DOTS) and the lengthy treatment protocols. Together, these factors promote nonadherence, contributing to relapse and establishment of drug-resistant Mtb strains. Although antibiotic treatment of drugsusceptible $M t b$ is generally effective, drug-resistant TB has a treatment efficacy below $50 \%$ and can, in a proportion, develop into progressive, untreatable disease. Other immune compromising co-infections and/or co-morbidities require more complex prevention/treatment approaches, posing huge financial burdens to national health services. Novel TB treatment strategies, such as host-directed therapeutics, are required to complement pathogen-targeted approaches. Pre-clinical studies have highlighted promising candidates that enhance endogenous pathways and/or limit destructive host responses. This review discusses promising pre-clinical candidates and forerunning compounds at advanced stages of clinical investigation in TB host-directed therapeutic (HDT) efficacy trials. Such approaches are rationalized to improve outcome in TB and shorten treatment strategies.
\end{abstract}

Mucosal Immunology (2020) 13:190-204; https://doi.org/10.1038/s41385-019-0226-5

\section{INTRODUCTION}

Tuberculosis (TB) remains the leading cause of death by infection worldwide. ${ }^{1}$ Despite introduction of directly observed treatment short-course (DOTS), the reduction in the global TB burden has been modest. The crisis is exacerbated by coinfections and co-morbidities, drug-resistant (DR) Mycobacterium tuberculosis $(M t b)$ strains and a rise in the reservoir of latent infection. Host immune status plays a determining role in TB disease outcome. It is also well-known that $M t b$ itself imposes several evasion strategies and prompts the host to elicit an immune response that favours its persistence. Adjunctive treatments aimed at "re-educating" the immune system are realistic alternative approaches to tailor host anti-TB responses. The use of host-directed therapeutics (HDTs) is intended to increase the success of TB treatment by immunomodulation and/or immune augmentation. Here, immunomodulation alludes to down-regulating non-productive inflammation and modifying the immune response. In contrast, immune augmentation is considered in the framework of synergizing with anti-TB treatment regimens of drug susceptible (DS)- and DR-TB to improve long-term outcome and promote cure.

HDTs are, therefore, considered crucial to achieving the 2035 World Health Organization (WHO) End TB goals. ${ }^{2}$ Repurposed compounds are more likely to be investigated in human clinical trials. In this regard, prior safety and regulatory approval increases the likelihood of fast-tracked implementation of drugs as appropriate immune response modifiers. Here we introduce HDT agents at advanced testing stages and highlight promising candidates for future HDT evaluations. These candidates may reveal favourable clinical outcomes and translate into useful adjunctive treatment strategies in our fight against TB.

\section{HOST IMMUNE CHARACTERISTICS OF TB}

TB disease is perceived as a paradigm of host immune failure. In contrast, latency is considered a proxy of immunological control of $M t b$ infection. There is, however, no clear consensus of what constitutes clinically protective immunity. First-line innate immune defences play a central role in TB pathogenesis, albeit insufficient to clear infection. For this reason, T-helper (TH)-1 and CD8 T-cell adaptive responses are considered crucial for effective anti-TB immunity. ${ }^{3,4}$ Conversely, type-I interferon (IFN) and typical TH2 responses are associated with disease progression, contributing to disease susceptibility. Additionally, regulatory T cells (Tregs) may inhibit protective immunity. 5 ,6 Theoretically, each of these pathways constitutes potential and 'druggable' targets. However, this notion is complicated by the complex course of progressive TB disease, including stages such as initial infection, protracted latency and overt disease ${ }^{7,8}$ Furthermore, other factors such as genetic diversity and co-morbidities (e.g. type-2 diabetes and HIV infection) also have a role to play.

A more recent concept is that TB represents a dynamic spectrum of mycobacteria at varying states of replication, ${ }^{7}$ highlighting the importance of immunotherapeutics treating the full TB spectrum. Realistically, a single immunotherapeutic agent is unlikely to be effective in the full TB spectrum. This has led to the concept of precision medicine approaches, since patient groups are likely to vary in their need for HDTs directed

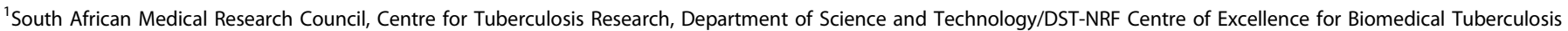
Research, Division of Molecular Biology and Human Genetics, Faculty of Medicine and Health Sciences, Stellenbosch University, Cape Town, South Africa Correspondence: N. Du Plessis (nelita@sun.ac.za)
} 
at immunomodulation and/or immune augmentation. For example, the treatment requirements from HDTs for individuals with advanced TB disease or even post-TB lung disorders are likely to differ considerably from those required for latently infected community members or healthy contacts of TB patients.

The National Institutes of Health $(\mathrm{NIH})$ clinicaltrials.gov resource database of privately and publicly funded human clinical trials lists investigations on adjunct therapies for various forms of TB. A literary search of human clinical trials, animal model studies and preliminary in vitro cohort studies was performed to identify current research highlighting repurposed drugs, HDTs and adjunctive candidates for TB treatment. Many of these have confirmed effective therapeutic manipulation of host immunity against $M t b$ and realignment of the response to support immune protection. Within the context of repurposed drugs, we summarize the four main mechanisms by which these adjunctive therapies are thought to improve outcome in TB (Fig. 1); namely, (1) mediating non-productive inflammation and inflammationinduced tissue pathology to improve lung function/integrity, (2) enhance host immune response efficacy and strengthen immune and memory responses, (3) enhance host bactericidal mechanisms, macrophage-mediated Mtb killing and reducing bacilli growth, and (4) disrupting and penetrating the granuloma to expose $M t b$ bacilli to anti-TB treatment.

\section{PROMISING TB HDT CANDIDATES TESTED IN PRE-CLINICAL AND HUMAN CLINICAL TRIALS}

Eicosanoid modulating drugs

Catabolism of arachidonic acid by cyclooxygenase (COX) enzymes produces prostaglandins, whereas lipoxygenase (LOX) metabolism yields leukotrienes. ${ }^{9}$ These eicosanoid products serve as signalling molecules, modulating inflammation and cell death. A delicate balance in eicosanoid levels is crucial for Mtb control and regulating the production of pro-inflammatory cytokines, such as tumour necrosis factor (TNF)-a (which plays a dual role in protection and exacerbated pathology in TB). Several modulators of the arachidonic acid pathway have been evaluated in humans as TB HDT.

Non-steroidal anti-inflammatory drugs (NSAID). Emerging evidence shows that heightened levels of prostaglandins at late stages of $M t b$ infection ( $>45$ days post-infection in mice) promote TB disease progression by down-regulating cellmediated immunity. ${ }^{10}$ NSAID are commonly prescribed analgesic

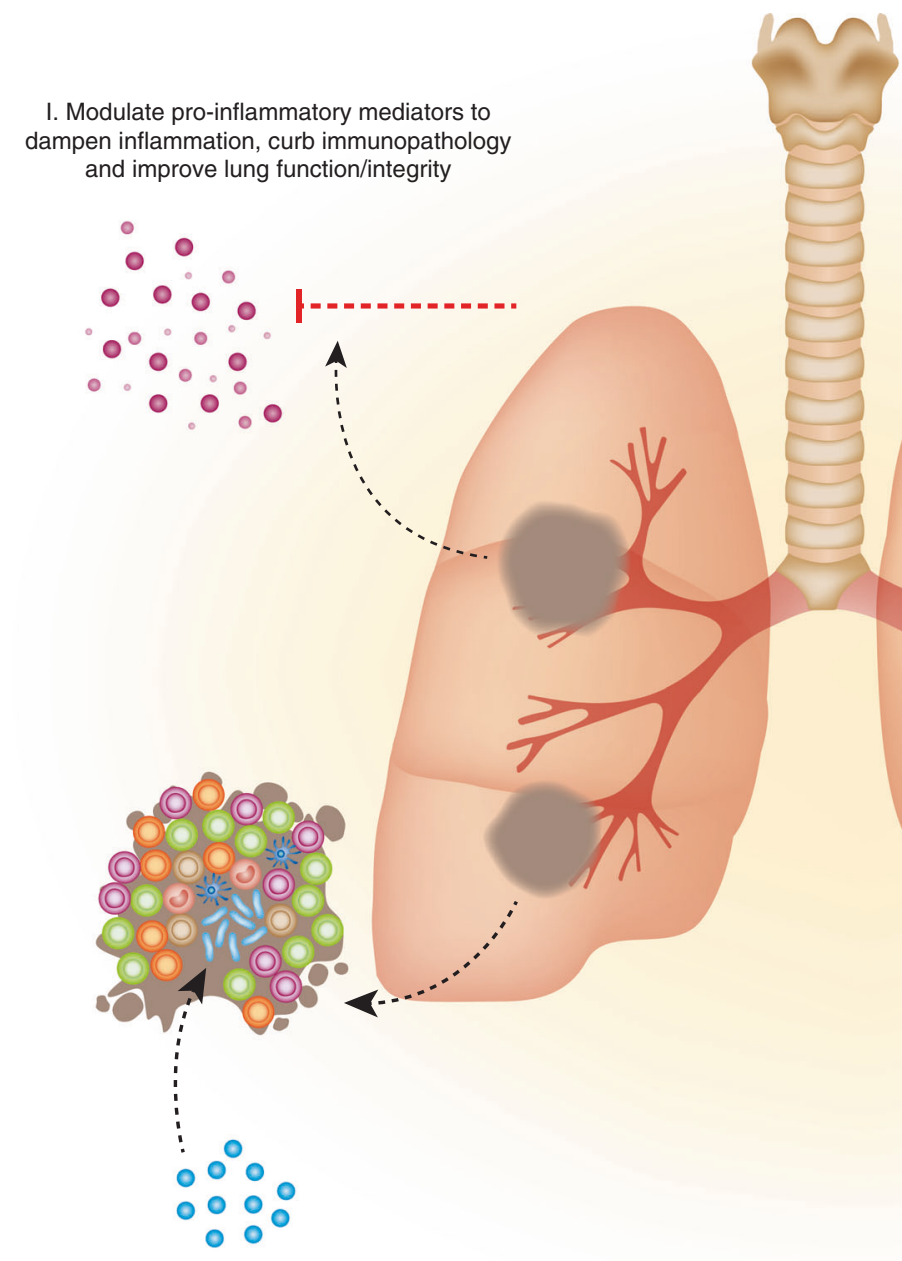

IV. Disrupt structure and penetrate granuloma to expose Mtb to anti-TB treatment
II. Enhance immune reponse efficacy, strengthen immune and memory responses

Fig. 1 Main mechanisms by which repurposed, adjunctive compounds improve outcome in TB; I modulate inflammatory pathways and proinflammatory mediators to dampen inflammation and inflammation-induced tissue pathology and improve lung function/integrity, II enhance host immune response efficacy and strengthen immune and memory responses, III enhance host bactericidal mechanisms, macrophage-mediated Mtb killing and reducing bacilli growth, and IV disrupting and penetrating the granuloma to expose Mtb bacilli to antiTB treatment. 
and anti-inflammatory medications worldwide and have shown promise as HDT in several pre-clinical studies. ${ }^{11-13}$ NSAID exert their effects by inhibiting COX activity, thereby interrupting formation of pro-inflammatory and immunosuppressive mediators such as prostaglandins and leukotrienes. ${ }^{9,14}$ Thus, the rationale for use of NSAID as HDT encompasses the inhibition of proinflammatory COX enzymes to attenuate excessive inflammation-induced tissue pathology and to improve host bactericidal mechanisms in individuals with active TB disease.

Resultantly, clinical trials have been initiated to assess the safety and efficacy of NSAID as adjunctive treatment of DS- and DR-TB (NCT02781909; NCT03092817; NCT02060006). For example, aspirin was investigated as an HDT candidate in a randomized trial during early TB treatment with dexamethasone in adult TB meningitis. Findings suggest that aspirin reduces new brain infarcts and related deaths through a mechanism involving inhibition of thromboxane-A2 and increased levels of protectins. ${ }^{15}$ Earlier trials also demonstrated reduced TB meningitis-associated strokes and mortality (Table 1). ${ }^{16}$ New generation NSAID selectively inhibiting the COX-2 enzyme are associated with less side-effects and gastrointestinal complications. Celecoxib and etoricoxib are currently undergoing evaluation in phase-I trials for safety and bactericidal activity in healthy volunteers and for efficacy as HDT in DS-TB (NCT02602509; NCT02503839; Table 1). Daily meloxicam, another selective COX-2 inhibitor, is currently under investigation in a randomized control trial, for its ability to prevent development and severity of paradoxical TB immune reconstitution inflammatory syndrome (TB-IRIS) (NCT02060006). The WHO recommends routine inclusion of NSAID as an adjunctive therapy to the standard TB treatment regimen to reduce antibiotic-related joint pain. However, the use of NSAID as preventative treatment remains unclear. Compared with nonusers, use of traditional NSAID was associated with an increased risk of TB in an unadjusted analysis of a population-based study. ${ }^{17}$ Results from carefully controlled trials should provide more conclusive findings on the effect of these inexpensive and widely available compounds on TB treatment outcomes.

Lipoxygenase inhibitors. Eicosanoids were previously suggested as targets for therapeutic exclusion in TB. However, data demonstrate the protective role of prostaglandin E (PGE)-2 during early infection, either by direct supplementation or via inhibition of 5-Lipoxygenase (5-LOX). Inhibiting 5-LOX has been linked to restricted lung pathology, lower type-I IFN production, reduced Mtb replication and greater survival rates in a TB-susceptible murine model $^{18}$; thus rationalizing the prospective use as adjunctive therapy to improve TB outcome. Accordingly, individuals with latent TB, who fail to develop active TB disease, display balanced levels of PGE-2 and lipoxins. ${ }^{18}$ Lipoxins also negatively regulate protective $\mathrm{TH} 1$ responses. This was demonstrated by increased IFN- $\gamma$, interleukin (IL)- 12 and nitric oxide synthase (NOS)2 mRNA levels and reduced mycobacterial burden in 5-LOXdeficient mice. ${ }^{19}$ At present, no trials evaluating 5-LOX inhibitors as HDT complementing standard TB treatment are registered on the clinicaltrials.gov resource database. The 5-LOX inhibitor, zileuton, is however, approved for treating asthma which could be repurposed as TB HDT and tested to elucidate whether modulation of this pathway improves TB treatment outcomes.

\section{Inflammatory modulators}

Corticosteroids. Corticosteroids have been employed as adjunctive therapy for a range of inflammatory conditions and disease states, including bacterial and viral meningitis, pneumonia and sepsis. $^{20,21}$ In TB, hyper-activation of the inflammatory response often results in tissue pathology and oedema, leading to tissue dysfunction and chronic inflammation. The rationale for using anti-inflammatory corticosteroids as adjunctive treatment of active TB disease mechanistically involves modulation of inflammatory and apoptotic gene transcription pathways. ${ }^{22}$ This occurs by binding to intracellular receptors and modulating gene transcription in target tissues, thus modulating inflammatory mediator function, suppressing the humoral immune response and inhibiting leucocyte infiltration to the site of disease. ${ }^{23,24}$ These effects are thought to reduce chronic, non-productive inflammation and favour the host antimicrobial response.

Corticosteroids as immunoadjuvants to standard TB treatment have proven useful in several studies, including an HIV/TB coinfection framework. Supporting evidence demonstrates improved lung radiological lesions, earlier symptomatic improvement and reduced morbidity in severe disease. ${ }^{25-28}$ In particular, trials testing the efficacy of adjunctive dexamethasone treatment on the risk of death or disability in TB meningitis demonstrated improved patient survival rate (Table 1). ${ }^{29}$ Other phase-III and IV multicentre trials, investigating survival and disability outcomes following dexamethasone adjunctive treatment of TB meningitis, are underway (NCT03100786; NCT03092817; NCT02588196; Table 1). Similarly, prednisolone for treating TB pericarditis in HIV infection was investigated in a phase-III trial (NCT00810849; Table 1). Results indicate no significant effect on the combined outcome of death, cardiac tamponade or constrictive pericarditis, although prednisolone did reduce incidences of pericardial constriction and hospitalization. ${ }^{30}$

Importantly, since data suggest that the effects and benefits of corticosteroid adjunctive therapy are organ specific, its use in extrapulmonary TB requires careful consideration on a casespecific basis. Meta-analyses refute long-term treatment efficacy of corticosteroids. In fact, studies involving high-dose corticosteroid treatment observed an increased risk of side effects. ${ }^{31}$ Lowdose trials, however, appear to circumvent such consequences, while maintaining favourable clinical outcomes in pulmonary TB (PTB) disease. 25,31,32 Taken together, it is evident that more investigation is needed to establish conclusive outcomes for the risks and benefits of corticosteroids as adjunctive therapy for advanced TB disease.

Phosphodiesterase inhibitors. Phosphodiesterase inhibitors (PDE-i) are small-molecule inhibitors that reduce inflammation by increasing intracellular cyclic adenosine monophosphate (CAMP) and CGMP. ${ }^{33}$ Altogether, the anti-inflammatory effects of PDE-i serve to modulate chronic inflammation and cytokine storms associated with infectious disease, while improving antibacterial responses and reducing bacillary load. This suggests relevance, not only during active TB disease, but also in clearing non-productive inflammation for conditions such as TB-IRIS and extrapulmonary TB.

Several selective PDE-i have shown promise as HDT candidates in TB animal models. Inhibitors of PDE-3 and PDE-5, cilostazol and sildenafil respectively, accelerated bacterial clearance and lung sterilization in murine TB. ${ }^{34}$ The PDE-4-i, roflumilast has also shown promise as an effective HDT in a TB mouse model when used with isoniazid. Supporting evidence illustrated reduced TNF-a production, thwarted neutrophil recruitment and reduced lung bacillary burden. ${ }^{35}$ Similar findings were reported for another selective PDE-4-i, CC-11050, in a TB rabbit model. ${ }^{36}$ Analogues of thalidomide, such as CC-3052, have also shown to possess similar PDE-4-i properties and demonstrated potential as TB HDT by reducing lung pathology and inflammation. ${ }^{37}$ These promising pre-clinical screenings of PDE-i have led to safety and efficacy testing of adjunctive CC-11050 with the standard 6-month multidrug therapy. This phase-II open-label human clinical trial is currently recruiting South African TB patients (NCT02968927; Table 1). Pending outcome of these results, several other members of the PDE-i family represent attractive HDT candidates. These include PDE-5-i, shown to reverse the host immunosuppressive effects of regulatory immune cells such as myeloid-derived suppressor cells (MDSC) in cancer. ${ }^{38}$ 


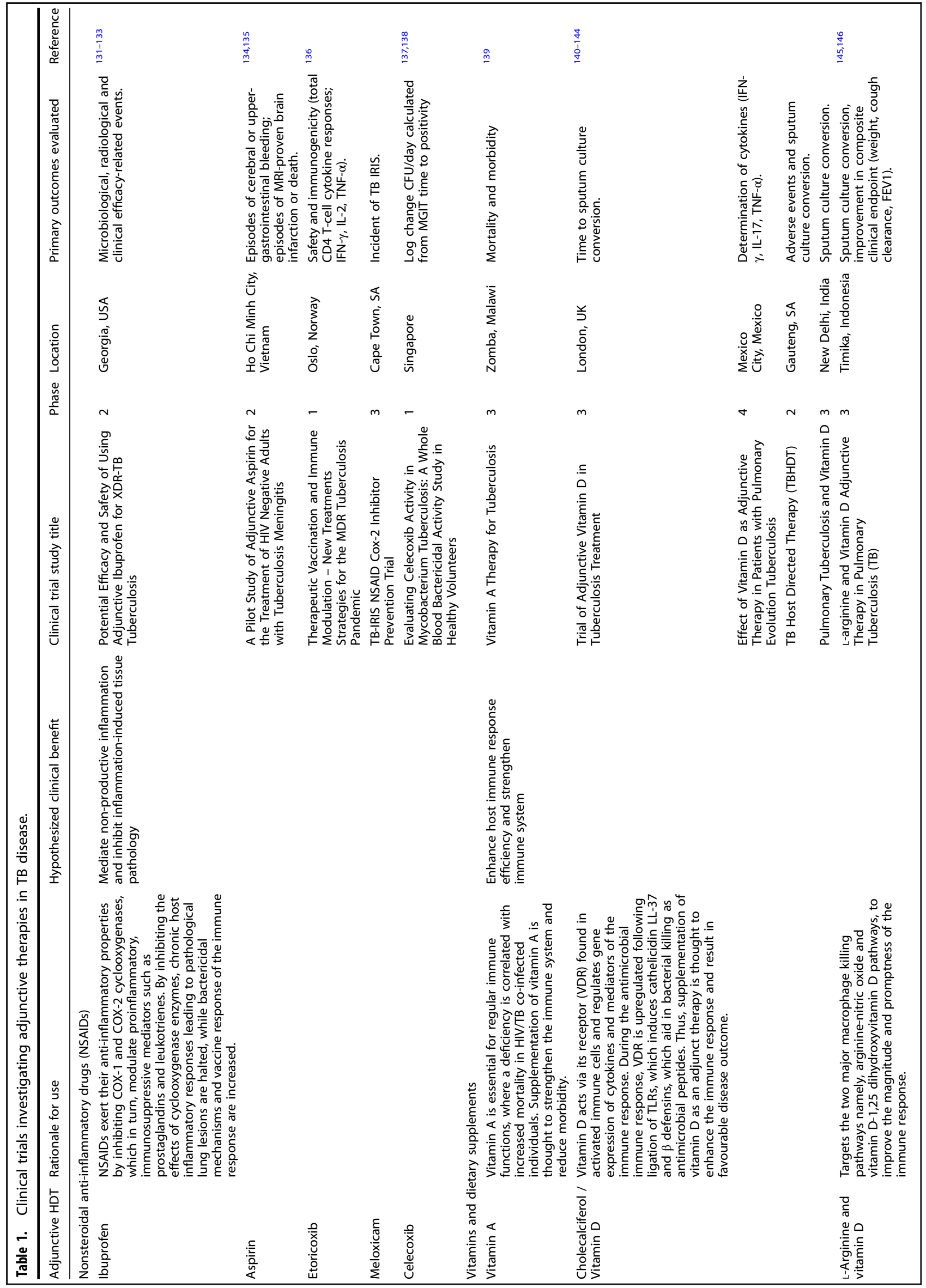




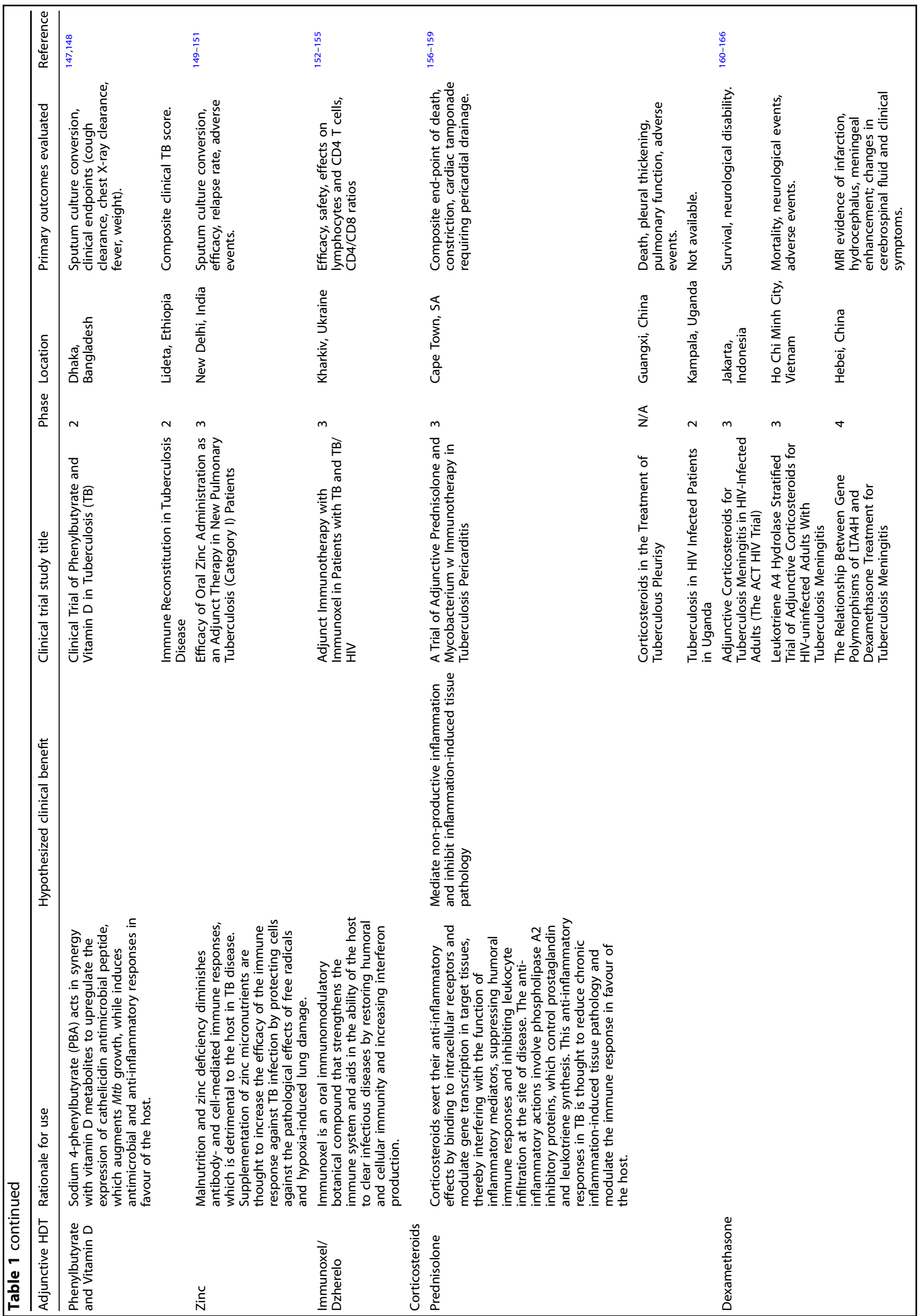




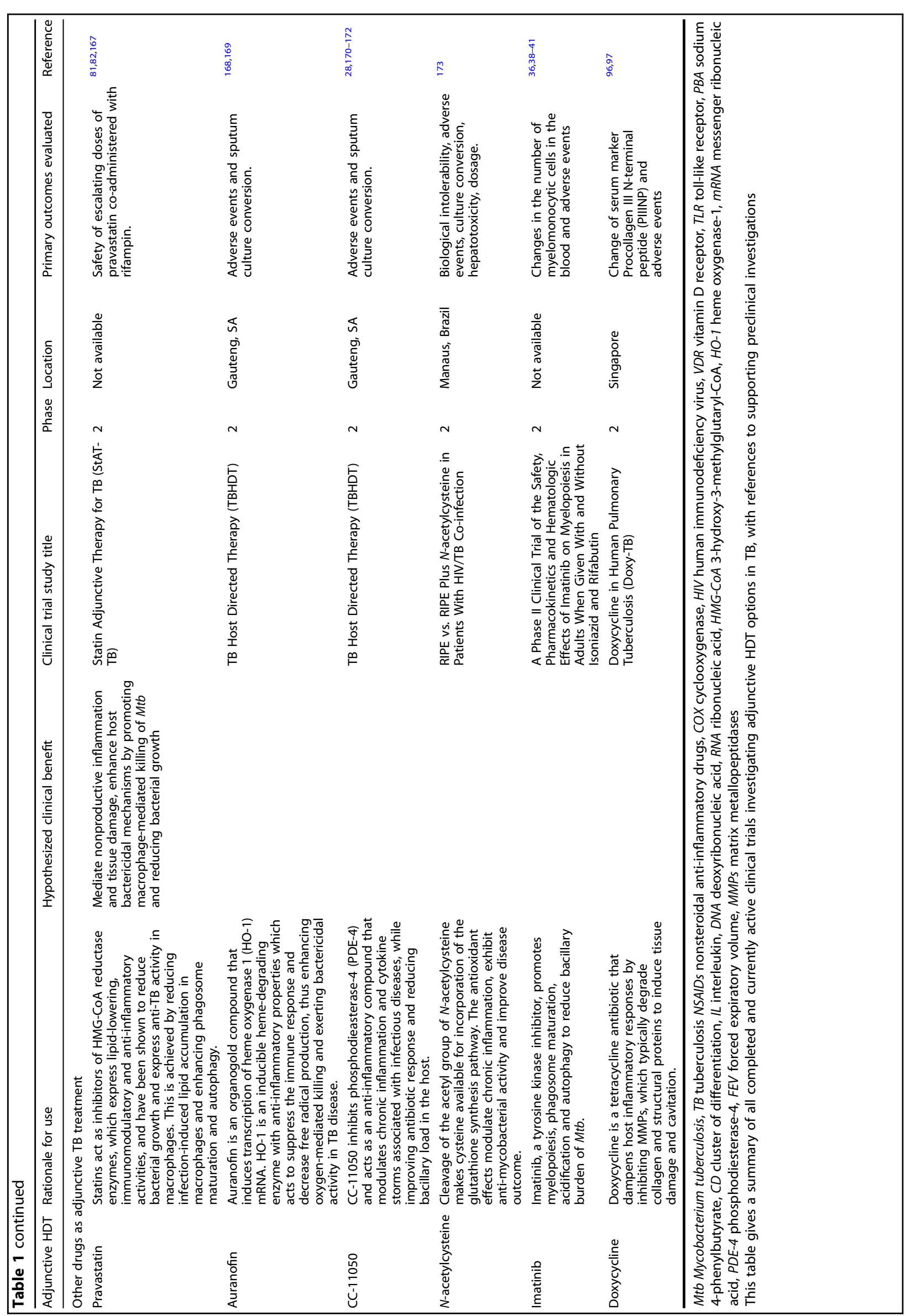


$\mathrm{N}$-Acetylcysteine (NAC). NAC is an L-cysteine prodrug, which replenishes levels of the antioxidant glutathione by making cysteine available for incorporation into the glutathione synthesis pathway. NAC, often prescribed to patients with chronic pulmonary disease, has mucolytic and antioxidant activities, with the capacity to modulate inflammation. ${ }^{39}$ In vitro data rationalizing improved outcome in TB indicate a dose-dependent NACmediated reduction in Mtb growth and metabolic activity. This occurs by suppressing the host oxidative response, along with direct anti-mycobacterial affects. ${ }^{40,41}$ Therefore, beneficial effects of NAC as HDT is not limited to use in symptomatic TB disease and post-TB lung disease, but also to clear Mtb in healthy latently infected individuals.

NAC has subsequently been tested in a prospective randomized control trial, demonstrating significantly faster sputum conversion with improved lung pathology in TB patients receiving daily NAC treatment during the intensive phase of DOTS. ${ }^{42}$ Additionally, NAC has a hepatoprotective effect on liver injury during TB treatment (Table 1), ${ }^{43,44}$ and a murine macrophage model has illustrated the ability of NAC to potentiate the efficacy of TB chemotherapy, specifically in combination with isoniazid. ${ }^{46}$ Together, these data provide promising outlooks for NAC as an adjunctive therapy to synergize with current therapies and improve outcome in TB. Currently, a phase 2 randomized trial is investigating the tolerability and treatment outcome of daily adjunctive NAC for 2 months in conjunction with the standard 6-month TB treatment regimen in Brazil (NCT03281226). Outcomes from ongoing studies should provide greater insight and justification for further trials investigating concomitant NAC for treatment of multi-drugresistant (MDR)-TB patients (Table 1).

Tyrosine kinase inhibitors

Another avenue for treating MDR-TB and HIV/TB co-infection includes the use of tyrosine kinase inhibitors as HDT. Imatinib is a tyrosine kinase inhibitor typically employed for treatment of cancers, more specifically, chronic myelogenous leukaemia. In the context of Mtb infection, beneficial outcomes of imatinib are associated with reducing bacillary burden by promoting myelopoiesis, phagosome maturation and acidification, and autophagy. ${ }^{45,47-49}$ Findings have illustrated that imatinib as an adjunctive therapy with first-line anti-TB drugs has synergistic therapeutic effects. ${ }^{48,49}$ A study by Steiger and colleagues in 2016 showed that imatinib induced lysosome acidification and antimicrobial activity against $M$. bovis in human macrophages treated with glucocorticoids. Notably, these effects were exhibited without reversing the anti-inflammatory effects of glucocorticoids. ${ }^{50}$ A clinical trial (NCT03891901; Table 1) is scheduled to roll out soon, which aims to evaluate safety, pharmacokinetics and effects of imatinib on myelopoiesis in adults, as a potential adjunctive therapy with an antimicrobial regimen for DS-TB.

Other tyrosine kinase inhibitors have shown potential in in vitro and murine studies. One candidate specifically, geftinib, an FDAapproved inhibitor of epidermal growth factor receptor (EGFR) tyrosine kinase, has shown promise in both acute and chronic Mtb infection, by augmenting TH1 immunity and reducing bacterial load. ${ }^{51}$ Tyrosine kinase inhibitors that have been studied extensively in tumour models, such as nilotinib, are now showing promising pharmacological expansion of protective innate immunity to mycobacterial infections. ${ }^{52}$ These compounds are attractive candidates for testing as a prophylactic anti-TB regimen in highrisk communities.

\section{Antihyperglycaemic drugs}

Poor glycaemic control is a risk factor for TB disease onset, mortality, treatment failure and relapse. ${ }^{53,54}$ In this regard, metformin (class: biguanide) treatment improves glucose control in diabetic patients and restores dysfunctional immunity associated with hyperglycaemia. ${ }^{55-57}$ In the context of TB, the immunomodulatory effects of metformin have been shown to promote macrophage autophagy by activating the expression of AMP-activated protein kinase (AMPK) and reactive oxygen species (ROS) production. Altogether these effects inhibit Mtb growth, reduce inflammation and prevent lung damage. ${ }^{58}$ These findings have promoted metformin as a candidate for therapeutic prevention and adjunctive treatment approaches in TB. ${ }^{55}$

In vitro and in vivo murine studies have further shown that metformin treatment reduces inflammation in TB by promoting expansion of anti-inflammatory cell types, particularly alternatively activated macrophages. ${ }^{59-62}$ A number of pre-clinical studies further demonstrated that metformin synergizes with the antimicrobial properties of rifampicin and reduces intracellular Mtb growth. This occurs in an AMPK-dependant manner, through inhibition of pro-inflammatory cell proliferation, thereby reducing disease severity. ${ }^{63,64}$ Other in vitro and in vivo findings show that metformin promotes ROS production, required for fusion of the phagosome-lysosome complex to aid in phagocytosis- and autophagy-induced killing of $M t b .{ }^{64,65}$ Metformin also has a direct effect on the bacterial respiratory chain complex, which plays an important role in bacterial persistence and tolerance. ${ }^{66}$ Retrospective evaluation of clinical trial data demonstrated that metformin treatment of type-II diabetics with TB is associated with fewer lung cavities, lower proportion of individuals with advanced disease and improved sputum culture conversion rate 2 months post-treatment initiation. ${ }^{58,64,67}$ Retrospective analyses also showed that type-II diabetics receiving metformin treatment have a lower TB risk profile compared to those using sulfonylureas. ${ }^{68}$ Interestingly, diabetics on metformin treatment have a lower chance of having latent $M t b$ infection (LTBI), as measured by a positive T-Spot TB test. ${ }^{64}$

Most clinical testing has been limited to evaluating the effects of metformin on diabetic TB patients. This begs the question of whether these same properties translate to non-diabetic TB patients in a clinical setting. Pre-clinical evidence of a recent in vitro study showed metformin-mediated modulation of cellular metabolism, immune function and gene transcription involved in innate immune responses to $M t b$ in heathy subjects. ${ }^{69}$ Moreover, metformin has illustrated beneficial effects for non-diabetic indications such as obesity, polycystic ovary syndrome and Alzheimer's disease. ${ }^{70}$ The first prospective trial testing the addition of metformin to DOTS, the 'Metformin for TB/HIV Hostdirected Therapy' (METHOD) trial, is currently in planning and will evaluate the proportion of smear negative TB patients at month two post-treatment. This trial will also test the efficacy of metformin on lung function, severity of lung involvement and HIV viral load (R34-Al124826-01).

While the TB community is anxiously awaiting results from clinical trials testing these and other drugs with anti-inflammatory or antihyperglycemic properties, it remains important to carefully consider and assess ideal dosages to prevent excessive antiinflammatory responses, which too may favour Mtb proliferation.

Vitamins and biologics

Vitamins are furthest along the pipeline of TB HDT testing in human clinical trials (Table 1). This is likely attributed to the relative ease of accessibility and low risks associated with vitamin supplementation. Vitamins are essential for regular immune function and deficiencies have been implicated in a range of disease states. As such, vitamin supplementation may be applicable as an adjunctive therapy to the standard of care to improve outcome to TB disease. On the other hand, perhaps vitamin supplementation may pose as a preventative strategy to strengthen the immune system and prevent progressive onset of disease.

Vitamin D (vitD), acting via its vitD receptor (VDR), regulates gene expression of cytokines and immune mediators in activated cells. In the antimicrobial immune response, VDR is upregulated 
following ligation of TLRs, which induces antimicrobial peptides such as cathelicidins and defensins. Thus, vitD as an adjunctive therapy may enhance the immune response and favourable disease outcome in TB. At least 22 current and completed trials of vitD as TB HDT are listed on the clinicaltrials.gov database. Inconsistencies in trial outcomes have, however, impeded interpretation of HDT efficacy. While some studies on vitD supplementation during TB treatment demonstrate clinical and radiological involvement in patients with vitD deficiency (Table 1), others fail to show any advantage on TB outcomes (Table 1)..$^{71,72}$ At a pre-clinical level, the protective effects of vitD has been linked to enhanced innate immune production of ROS, IL-1 $1 \beta$, IFN- $\gamma$ and cathelicidin, ${ }^{73,74}$ while positive trials have shown a reduction in inflammatory mediators including matrix metalloproteinases (MMPs) (Table 1). It is believed that contrasting trial outcomes reflect variations in vitD administration and dosage, differing levels of endogenous baseline vitD, genetic differences in the vitD receptor, underpowered cohorts and variations in sunlight exposure at trial locations.

Vitamin A (vitA) deficiency has also been associated with incident TB and correlated with increased mortality in HIV/TB coinfected individuals. VitA supplementation is thought to strengthen the immune system and reduce mortality; however, information on vitA supplementation in conjunction with TB treatment has been inconsistent. The handful of completed casecontrol studies investigating vitA supplementation during TB treatment mainly report findings as part of a dietary multivitamin supplement. ${ }^{75}$ Therefore, evidence of the direct benefit of vitA has been weak, at best demonstrating modest improvements in the weight of TB patients. ${ }^{76}$ VitA supplementation with zinc yielded similar results ${ }^{75}$ (Table 1). A more recent case control study, nested within a longitudinal TB household contact study, showed that baseline vitA deficiency was associated with a tenfold increased risk of developing TB. ${ }^{77}$

Although the overall findings in the area of vitamins as TB HDT is promising, significant challenges exist that impede objective interpretation of data. This mainly stems from heterogeneous study design with discrepancies in nutritional/dietary intake and route of administration, amongst others. We propose that meticulous study design may overcome these challenges and may provide more conclusive data for the use of vitamin supplementation to improve TB outcome.

Sodium phenylbutyrate (PBA), a biological aromatic fatty acid, has been approved for treating various diseases, including urea cycle disorders, cancer, muscular dystrophy and Parkinson's disease. Its functions include inhibition of histone deacetylase and endoplasmic reticulum stress. ${ }^{78,79}$ Pre-clinical studies and clinical trial data have shown that PBA synergises with vitD to upregulate expression of the anti-mycobacterial peptide cathelicidin, restrict $M t b$ uptake, reduce $M t b$ intracellular growth in macrophages, upregulate chemokine secretion and induce autophagy. ${ }^{80,81}$ These benefits of PBA has been verified in a randomized controlled trial in Bangladesh, demonstrating its potential as TB HDT (Table 1).

Other immunomodulatory biologics include immune checkpoint inhibitors (ICls). The ICls currently attracting the most attention in TB include nivolumab and ipilimumab. Nivolumab is a monoclonal antibody targeted against programmed death (PD) 1 protein, while ipilimumab targets cytotoxic T-lymphocyteassociated antigen 4 (CTLA4). Typically, signalling via immune checkpoints inhibits $\mathrm{T}$ - and B-cell function. However, in the context of $\mathrm{TB}$, immune regulatory checkpoints are dysregulated and associated with T-cell exhaustion. ${ }^{82-86}$ Despite promising outcomes in animal and in vitro models, clinical use of ICls may favour progression to active TB disease, potentially attributed to excessive inflammation and focal necrosis. ${ }^{87}$ Such therapies thus require careful consideration regarding method, dose and timing of administration to minimise potential negative effects.
Cytokine modulation

Therapeutic modulation of immunity via cytokines is another method to support host defences. Cytokines play crucial roles in immune cell function and can theoretically serve as promising candidates for inclusion in adjunctive immunotherapies. This, however, is strictly dependent on role of a given cytokine in host immunity. Reducing excessive cytokine responses appears as a promising HDT strategy for individuals with active DR- and DS$\mathrm{TB}^{88,89}$ In contrast, boosting TH cytokine responses could serve as a feasible strategy for those with acute/recent Mtb infection. ${ }^{90,91}$

Cytokines may polarize the immune response in favour of host protection by strengthening immune and memory responses or by disrupting and penetrating the granuloma to expose $M t b$ bacilli to anti-TB treatment (Table 2; Fig. 1). The hallmark TH1 cytokines, namely IFN- $\gamma$, IL-2, IL-12 and GM-CSF, have been highlighted as recombinant therapeutics in adjunctive HDT trials. Additionally, the activity of TH2/immunosuppressive cytokines may be modulated as an alternative strategy ${ }^{92-96}$ (Table 2). A currently active, phase-II interventional trial of pascolizumab, an anti-IL4 monoclonal antibody, is being investigated in TB patients receiving standard treatment (NCT01638520; Table 1). As the TB community eagerly awaits this outcome, another randomized, placebo-controlled trial disappointingly demonstrated that adjunctive recombinant IL-2 immunotherapy in TB patients did not afford a statistically significant improvement in bacterial clearance as measured by culture conversion at months 1 and $2^{97}$ (Table 2).

Cytokine HDT approaches that have received considerable attention in TB involve IFN- $\gamma$ or modulation of TNF-a. In particular, aerosol administration of recombinant IFN- $\gamma-1 b$ as supplement to DOTS for patients with cavity PTB was evaluated in a phase-II trial. Results demonstrated favourable immunomodulation by reducing inflammatory cytokines at the site of disease and accelerated Mtb sputum clearance (Table 2). ${ }^{93}$ More recent pre-clinical data have however illustrated the propensity for exacerbated lung infection and deleterious effects of increased IFN- $\gamma$ production by CD4 T cells in murine models. ${ }^{98}$ While TNF-a stimulates monocytes/ macrophages and maintains granuloma integrity, high levels may exacerbate pathology. Thus, approaches that decrease TNF-a have been favoured with the rationale of restricting pathology or destabilizing fibrotic granulomas to improve drug penetration ${ }^{99}$ (Table 2). TNF-a-blockers routinely used for treating inflammatory bowel disease and arthritis (such as etanercept) demonstrated some benefit in TB, while TNF-a-antibodies (such as infliximab and adalimumab) have shown success in advanced TB disease. ${ }^{101-102}$ In contrast, a recent meta-analysis indicated that the risk of TB may be significantly increased in patients treated with TNF-a antagonists, and may be evoking more harm than good in majority of patients. ${ }^{103}$ Therefore, despite some promising therapeutic outcomes, the use of IFN- $\gamma$ and TNF- $a$ modulating agents remains controversial, ultimately due to their interactions being both synergistic and antagonistic.

Considering their involvement in highly complex networks, the therapeutic impact of cytokines is often challenging to predict. This reiterates the importance of critically evaluating dosage systems to ensure optimal benefit to recipients. Additionally, despite promising outcomes of some cytokine-based therapies, employment of such strategies may be restricted by high cost, potential toxicity and role in immunopathology. ${ }^{104}$ Lastly, it has become increasingly evident that single-cytokine HDTs are often inadequate during the initial phase of therapy, and thus requires further exploration for combinatory cytokine therapy options.

Statins and other drugs

Statins are well-known for their lipid-lowering, immunomodulatory and anti-inflammatory activities. These effects are achieved via inhibition of HMG-CoA ( $\beta$-Hydroxy $\beta$-methylglutaryl-CoA) reductase enzymes, which are essential in lipid metabolism and 
Table 2. The role of cytokines in TB disease and evidence to support their therapeutic intervention and outcome as TB HDT strategies.

\begin{tabular}{|c|c|c|c|}
\hline Cytokine & Role in TB disease & $\begin{array}{l}\text { Therapeutic intervention and outcome in TB } \\
\text { disease }\end{array}$ & Reference \\
\hline INF- $\gamma$ & $\begin{array}{l}\text { Activates macrophages and DCs, promotes cell } \\
\text { proliferation, apoptosis, cell adhesion and } \\
\text { bacterial killing (through phagocytosis and } \\
\text { reactive nitrogen and oxygen intermediates). }\end{array}$ & $\begin{array}{l}\text { Aerosol administration of INF- } \gamma \text { improved } \\
\text { bacillary clearance and improved clinical } \\
\text { condition. Aerosol administration of INF- } \gamma \text { in } \\
\text { conjunction with anti-TB drugs cured MDR- } \\
\text { TB. Nebulized INF- } \gamma 1 \mathrm{~b} \text { and subcutaneous } \\
\text { injections of INF- } \gamma 1 \mathrm{~b} \text { alleviated disease } \\
\text { symptoms, although culture showed that } \\
\text { nebulized administration increased the } \\
\text { likelihood of negative smears at } 4 \text { weeks. } \\
\text { Supplementation of recombinant INF- } \gamma \\
\text { improves response to anti-TB drugs in } \\
\text { cavitary TB patients. }\end{array}$ & $75,174-177$ \\
\hline TNF- $\alpha$ & $\begin{array}{l}\text { Controls } M t b \text { infection and replication by } \\
\text { formation and maintenance of the granuloma; } \\
\text { by regulating macrophage activation, } \\
\text { phagocytosis, and nitrogen and oxygen } \\
\text { intermediates. }\end{array}$ & $\begin{array}{l}\text { TNF } \alpha \text { inhibition causes granuloma disruption } \\
\text { and bacillus reactivation to increase } M t b \\
\text { susceptibility to standard TB drugs; results in } \\
\text { rapid clearance of } M t b \text { from the lung and } \\
\text { altered inflammatory responses to benefit } \\
\text { the host in TB/HIV-1 co-infected individuals. }\end{array}$ & 178,179 \\
\hline GM-CSF & $\begin{array}{l}\text { Induces granulocyte and macrophage } \\
\text { proliferation and differentiation, stimulates } \\
\text { macrophage phagocytosis, increases } \\
\text { cytotoxicity and reactive nitrogen and oxygen } \\
\text { intermediates. }\end{array}$ & $\begin{array}{l}\text { Administration of GM-CSF resulted in } \\
\text { negative sputum culture conversion after } \\
8 \text { weeks. }\end{array}$ & \\
\hline TGF- $\beta^{*}$ & $\begin{array}{l}\text { Immunosuppressive cytokine, inhibits the Th1 } \\
\text { response during chronic infection. }\end{array}$ & $\begin{array}{l}\text { Suppression of TGF- } \beta \text { enhances resolution of } \\
\text { local Mtbinfection and associated } \\
\text { inflammatory responses, while decreasing } \\
\text { bacillary load in mouse models. }\end{array}$ & 180 \\
\hline VEGF* & $\begin{array}{l}\text { Angiogenic cytokine that promotes hypoxic } \\
\text { microenvironment. }\end{array}$ & $\begin{array}{l}\text { Neutralization or inhibition increases } \\
\text { efficiency of TB treatment regimens by } \\
\text { disrupting the granuloma thus promoting } \\
\text { drug penetration and Mtb killing in human, } \\
\text { rabbit and zebrafish models. }\end{array}$ & 181,182 \\
\hline IL-2 & $\begin{array}{l}\text { Aids proliferation of antigen-specific CD4+ and } \\
\text { CD8 }+ \text { T, activates the JAK-STAT signalling } \\
\text { pathway for gene transcription of cell growth } \\
\text { and survival genes. }\end{array}$ & $\begin{array}{l}\text { Recombinant IL-2 supplementation with } \\
\text { anti-TB drugs improved immunity status and } \\
\text { promoted sputum smear conversion to } \\
\text { negative, with reduced INF- } \gamma \text { production and } \\
\text { low skin response to Mtb antigens (response } \\
\text { is likely mediated by regulatory T cells). }\end{array}$ & 183 \\
\hline IL-4* & $\begin{array}{l}\text { Downregulates INF- } \gamma \text { production and mediates } \\
\text { cytotoxicity and fibrosis. }\end{array}$ & $\begin{array}{l}\text { Genetically deficient } I L-4^{-1-} \text { mice } \\
\text { successfully eradicated } M t b \text { infection } \\
\text { following reconstruction with recombinant } \\
\text { IL-4, and has the potential to be adjunctive } \\
\text { to standard TB regimens. }\end{array}$ & 184,185 \\
\hline IL-7* & $\begin{array}{l}\text { Enhances T-cell memory, upregulates IL-17 } \\
\text { production, downregulates TGF- } \beta \text {, aids in DC } \\
\text { activation. }\end{array}$ & $\begin{array}{l}\text { Mtb mouse models demonstrated increased } \\
\text { survival and bacilli clearance when } \\
\text { administered in conjunction with BCG } \\
\text { vaccine. }\end{array}$ & 186,187 \\
\hline IL-10* & $\begin{array}{l}\text { Immunoregulatory cytokine with Th2- } \\
\text { modulatory effects. }\end{array}$ & $\begin{array}{l}\text { Inhibition of IL-10 in conjunction with anti- } \\
\text { TB drugs effectively improved disease } \\
\text { outcome and drug efficiency. }\end{array}$ & 188 \\
\hline IL-12* & $\begin{array}{l}\text { Strong inducer of INF- } \gamma \text { production in antigen- } \\
\text { stimulated CD4+T cells, essential for protective } \\
\text { immune response to intracellular pathogens. }\end{array}$ & $\begin{array}{l}\text { Administration to } M t b \text {-infected mice } \\
\text { decreased viable bacilli load in lymphoid } \\
\text { organs. One case study provided evidence of } \\
\text { successful response to anti-TB drugs only } \\
\text { following treatment with IL-12 for } 3 \text { months } \\
\text { in a patient that was previously refractory to } \\
\text { anti-TB treatment. }\end{array}$ & 189,190 \\
\hline IL-15* & $\begin{array}{l}\text { Aids proliferation and survival of CD8 }+\mathrm{T} \text { cells, } \\
\text { strengthens immune memory. }\end{array}$ & $\begin{array}{l}\text { BCG-vaccinated IL-15 transgenic mice } \\
\text { displayed resistance against } M t b \text { infection, } \\
\text { thus acting as an immune adjuvant to } \\
\text { increase efficiency when administered with } \\
\text { BCG vaccination. }\end{array}$ & 186,191 \\
\hline IL-23* & $\begin{array}{l}\text { Inducer of INF- } \gamma \text { production and proliferation of } \\
\text { activated memory T cells. }\end{array}$ & $\begin{array}{l}\text { Vector-mediated intratracheal delivery in } \\
\text { mice reduced bacilli load and inflammation. }\end{array}$ & 192 \\
\hline
\end{tabular}




\begin{tabular}{|c|c|c|c|}
\hline Cytokine & Role in TB disease & $\begin{array}{l}\text { Therapeutic intervention and outcome in TB } \\
\text { disease }\end{array}$ & Reference \\
\hline IL-37* & $\begin{array}{l}\text { Antiinflammatory cytokine, broadly suppresses } \\
\text { innate and adaptive immunity. }\end{array}$ & $\begin{array}{l}\text { BCG-infected transgenic IL- } 37 \text { mice } \\
\text { displayed reduced bacilli load and tissue } \\
\text { damage in the lung, with reduced } \\
\text { frequencies of regulatory T cells and } \\
\text { Th17 cells. }\end{array}$ & 194 \\
\hline \multicolumn{4}{|c|}{ Cytokine modulators } \\
\hline RhIL-2 & $\begin{array}{l}\text { Cytokine adjunctive therapy is thought to } \\
\text { restore the immune response and modulate the } \\
\text { immunologic status in favour of the host by } \\
\text { promoting CD } 4+\text { and CD }+ \text { T-cell proliferation, } \\
\text { and by activating gene transcription pathways } \\
\text { of cell growth and cell survival genes. }\end{array}$ & $\begin{array}{l}\text { Study of adjunctive recombinant human } \\
\text { interleukin-2 therapy in patients with MDR- } \\
\text { TB }\end{array}$ & $74,183,195,196$ \\
\hline
\end{tabular}

inflammatory pathways. Since the lipid-rich macrophage is a favourable environment for $M t b$ persistence, statins reducing intracellular lipid accumulation thus limits bacterial growth. Moreover, statins enhance phagosome maturation and autophagy (Table 1). ${ }^{106-107}$ The StAT-TB trial is investigating the safety, tolerability and pharmacokinetics of pravastatin co-administered with standard TB treatment. The ability of pravastatin adjunctive therapy to shorten the time to sputum culture conversion and improve lung function will also be tested in a second phase of this trial (NCT03456102; Table 1). Of particular interest is the drug - drug interactions between statins and anti-TB drugs such as rifampicin and isoniazid. ${ }^{108}$ The associated adverse events such as myopathy and rhabdomyolysis are often associated with nonadherence and unsuccessful treatment, making it particularly important to select statins with no known drug interactions with TB antibiotics.

Another drug, auranofin, is an organogold compound that induces transcription of heme-oxygenase-1. This inducible hemedegrading enzyme exerts anti-inflammatory properties and decreases free radical production, while enhancing oxygenmediated killing and bactericidal activity in TB disease. A trial in South Africa is currently recruiting TB patients to test the safety and efficacy of auranofin as an adjunctive TB HDT (NCT02968927; Table 1).

Within the scope of cancer management, cell-based therapies are receiving growing interest. Indeed, such strategies may form a good template for HDTs in the TB field. As reviewed by Rao et al., in the context of MDR-TB, adoptive cell therapies and screening techniques could identify useful non-cross-reactive $M t b$ target-specific Tcell receptors (TCRs). These TCRs, in turn, may be transferred into recipient effectors (such as NK or T cells), theoretically giving rise to genetically modified therapeutic cellular products. ${ }^{109}$ Indeed, there has also been promising outcomes in MDR/extensively drugresistant (XDR)-TB patients receiving mesenchymal stromal cells (MSC) as a single infusion of bone marrow-derived autologous MSC. $^{110,111}$ These MSC are well-known for their safety, anti- inflammatory and immunomodulatory properties and may thus also be applicable to various forms of TB disease. ${ }^{109,112}$ Additionally, microRNAs (miRs) have been implicated as potential adjunctive HDTs to regulate immune responses in TB and improve outcome. This approach holds promise by using miR for repairing and replenishing miR stores or administering anti-miRs to inhibit rogue miR that may otherwise induce pathology. ${ }^{113,114}$ Although having solid theoretical foundation and promising outlooks for the future, cell-based therapies and miRs in the context of TB remains in its infancy, with much still to be uncovered.

Autophagy-activating compounds may represent promising adjunctive therapies against TB disease. A review by Paik et al. discusses autophagy mediators targeting VDR signalling, the AMPK pathway, sirtuin 1 activation and nuclear receptors. ${ }^{15}$ Autophagy-targeting small molecules have shown promise in the context of $M t b$ infection. In pre-clinical testing, gefitinib (targeting EGFR), fluoxetine (a serotonin reuptake inhibitor), baicalin (a herbal medicine targeting the $\mathrm{PI} 3 \mathrm{~K} / \mathrm{Akt} / \mathrm{mTOR}$ pathway) induce autophagy and enhance intracellular Mtb clearance. ${ }^{116,117}$ Indeed, antimicrobial drugs such as loperamide, verapamil and standard anti-TB drugs (such as INH and pyrazinamide) themselves promote autophagy and may work synergistically with autophagy-inducing small molecules as adjunctive therapy to standard treatment for TB patients. ${ }^{115,118-120}$

Although falling beyond the classification of HDT, antibiotics (such as doxycycline; NCT02774993; Table $1^{121,122}$ ) and vaccine strategies may modulate immunity, and have been proposed as potential adjunctives to the TB treatment regimen. There is evidence supporting beneficial effects of Bacille Calmette-Guérin (BCG) re-vaccination in adolescents and adults. Results indicate that BCG re-vaccination reduces the rate of sustained QuantiFERON (QFT) conversion and displays improved long-term innate or trained immunity and adaptive responses, thus leading to effective control of mycobacterial infection. ${ }^{123-129}$ A comprehensive review by Schaible et al. in 2017 evaluates strategies to improve vaccine efficacy against TB by targeting innate 
immunity. ${ }^{130}$ Here, they propose that short-term modulation of the local immune response to BCG vaccination may result in longterm protective immunity against $M t b$ infection. Examples of interventions for such modulation may involve regulating neutrophil, Treg and MDSC recruitment to the vaccination site, preventing disadvantageous cell death pathways, modulating vaccination-induced inflammatory responses, and regulating antiinflammatory cytokine profiles (e.g. IL-10). ${ }^{130}$ These strategies are aimed at processes that would otherwise negatively influence Tcell priming, function and proliferation upon vaccination; and many of which are in fact analogous to the rationale of HDT strategies discussed here.

\section{CONCLUSION}

While the outcome of some trials has been met with anticlimactic conclusions, emerging evidence outlined in this review suggests that the TB field is making steady progress in identifying beneficial HDTs across a broad range of drug classification and mechanistic activity. Building on these data, it is hoped that future investigations will translate into meaningful, effective clinical developments.

According to the therapies discussed in this review, we propose that certain HDTs will be of particular relevance to a specific TB infection/disease group. In this regard, we recommend the following HDT strategies to be most appropriate against active TB and associated forms of TB (such as, TB-IRIS, TB-induced pulmonary diseases and extrapulmonary TB): eicosanoid modulators (NSAID and lipoxygenase inhibitors), inflammatory mediators (corticosteroids and tyrosine kinase inhibitors), metformin, ICls, cytokine modulating therapy, statins, auranofin, cell-based therapies, miR and autophagy modulating drugs.

As TB preventative therapy, HDTs could, for example, alter bacillary cell entry or enhance anti-mycobacterial properties of lung phagocytes. Ideally, this would prevent infection, while also averting disease development in latently infected individuals. For TB contacts, those in high-exposure settings, recent Mtbinfected individuals and LTBI, we propose promising outcomes associated with host-strengthening preventative strategies, including vitamin supplementation, PBA (acting in synergy with vitD), NAC and BCG re-vaccination. We further propose HDTs to supplement new vaccines which may include: PDE-i to regulate effects of immunosuppressive subsets such as MDSC, ICls to modulate cell death pathways, cytokine therapy to regulate antiinflammatory cytokine profiles and perhaps eicosanoid and inflammatory mediators to modulate vaccine-induced inflammatory responses and potentiate vaccine-specific responsiveness and durability.

One of the major shortcomings of HDTs includes off-target and associated side effects. These drawbacks require further evaluation against the backdrop of other aspects such as storage stability, delivery method, formulation and timing of administration at different phases of Mtb infection and TB disease. In this context, several HDTs remain highly controversial and require more investigation into their potentially severe off-target and associated effects. Two major examples include, ICls and cytokine therapies. Although offering preventative HDTs to latently infected individuals remains a promising avenue, distinguishing latent infection from early active TB is however, challenging in high-exposure regions. Applicability of HDTs to MDR-TB, TB treatment shortening, TB/HIV and TB-derived lung diseases, although highlighted in some studies, have not been considered for all HDTs. This leaves much to be answered in the context of the TB spectrum. Furthermore, many theoretically sound approaches remain in their infancy in the TB field and require further investigation, hopefully showing promise and advancing to clinical trial status. Here we propose keeping a watchful eye on autophagy modulators, cell-based and miR therapies.
Considering the spectrum of TB disease formats and complexity of host immunity, adjunct HDT is unlikely to be efficient as a 'onesize-fits-all' approach. Even so, personalized medicine is also not feasible in high-burdened TB regions, making a case for a precision medicine approach, tailored to phenotypic disease groups. Therefore, developing biosignatures translating into an efficient, rapid, point-of-care pre-screening test is of great interest. In this way, immune profiling or patient stratification according to the degree of lung involvement or risk of disease relapse, will be a game changer for TB treatment strategies. The field remains inspired in the face of our ambitious goal to eradicate TB disease, and the even greater aspiration of preventing Mtb infection. These goals are hoped to be achieved through clever strategies involving multimodular approaches, including implementation of adjunct HDT as standard of care for TB patients.

\section{ACKNOWLEDGEMENTS}

The authors acknowledge the financial support from the European \& Developing Countries Clinical Trials Partnership (EDCTP; CDF1546).

\section{AUTHOR CONTRIBUTIONS}

The manuscript was written and developed by all three authors.

\section{ADDITIONAL INFORMATION}

Competing interests: The authors declare no competing interests.

Publisher's note Springer Nature remains neutral with regard to jurisdictional claims in published maps and institutional affiliations.

\section{REFERENCES}

1. WHO. Global tuberculosis report 2017. http://www.who.int/tb/publications/ global_report/en/. Accessed 28 April 2018.

2. WHO. WHO end TB strategy. http://www.who.int/tb/post2015_strategy/en/. Accessed 28 April 2018.

3. Huang, L. \& Russell, D. G. Protective immunity against tuberculosis: what does it look like and how do we find it? Curr. Opin. Immunol. 48, 44-50 (2017).

4. Maertzdorf, J. et al. Human gene expression profiles of susceptibility and resistance in tuberculosis. Genes Immun. 12, 15-22 (2011).

5. Turner, R. D. et al. Tuberculosis infectiousness and host susceptibility. J. Infect. Dis. 216, S636-S643 (2017).

6. Roberts, T., Beyers, N., Aguirre, A. \& Walzl, G. Immunosuppression during Active Tuberculosis Is Characterized by Decreased Interferon $-\gamma$ Production and CD25 Expression with Elevated Forkhead Box P3, Transforming Growth Factor- $\beta$, and Interleukin-4 mRNA Levels. J. Infect. Dis. 195, 870-878 (2007).

7. Malherbe, S. T. et al. Persisting positron emission tomography lesion activity and Mycobacterium tuberculosis mRNA after tuberculosis cure. Nat. Med. 22, 1094-1100 (2016).

8. Barry 3rd, C. E. et al. The spectrum of latent tuberculosis: rethinking the biology and intervention strategies. Nat. Rev. Microbiol. 7, 845-855 (2009).

9. Ricciotti, E. \& FitzGerald, G. A. Prostaglandins and Inflammation. Arterioscler. Thromb. Vasc. Biol. 31, 986-1000 (2011).

10. Moreno, J. R. et al. The role of prostaglandin E2 in the immunopathogenesis of experimental pulmonary tuberculosis. Immunology 106, 257-266 (2002).

11. Kroesen, V. M. et al. Non-steroidal anti-inflammatory drugs as host-directed therapy for tuberculosis: a systematic review. Front. Immunol. 8 1-9 (2017)

12. Dutta, N. K., Mazumdar, K., Dastidar, S. G. \& Park, J.-H. Activity of diclofenac used alone and in combination with streptomycin against Mycobacterium tuberculosis in mice. Int. J. Antimicrob. Agents 30, 336-340 (2007).

13. Byrne, S. T., Denkin, S. M. \& Zhang, Y. Aspirin and ibuprofen enhance pyrazinamide treatment of murine tuberculosis. J. Antimicrob. Chemother. 59, 313-316 (2007).

14. Maitra, A. et al. Repurposing drugs for treatment of tuberculosis: a role for nonsteroidal anti-inflammatory drugs. Br. Med. Bull. 118, 138-148 (2016).

15. Mai, N. T. et al. A randomised double blind placebo controlled phase 2 trial of adjunctive aspirin for tuberculous meningitis in HIV-uninfected adults. eLife 7, e33478 (2018). 
16. Misra, U. K. et al. A study of cytokines in tuberculous meningitis: clinical and MRI correlation. Neurosci. Lett. 483, 6-10 (2010).

17. $\mathrm{Wu}, \mathrm{C}$.-W. et al. Risk of incident active tuberculosis disease in patients treated with non-steroidal anti-inflammatory drugs: a population-based study. $B M C$ Pulm. Med. 17, 82-82 (2017).

18. Mayer-Barber, K. D. et al. Host-directed therapy of tuberculosis based on interleukin-1 and type I interferon crosstalk. Nature 511, 99-103 (2014).

19. Bafica, A. et al. Host control of Mycobacterium tuberculosis is regulated by 5 lipoxygenase-dependent lipoxin production. J. Clin. Invest. 115, 1601-1606 (2005).

20. Cohen, S. P. et al. Randomized, double-blind, comparative-effectiveness study comparing pulsed radiofrequency to steroid injections for occipital neuralgia or migraine with occipital nerve tenderness. Pain 156, 2585-2594 (2015).

21. de Gans, J. \& van de Beek, D. Dexamethasone in Adults with Bacterial Meningitis. N. Engl. J. Med. 347, 1549-1556 (2002).

22. Kadhiravan, T. \& Deepanjali, S. Role of corticosteroids in the treatment of tuberculosis: an evidence-based update. Indian J. Chest Dis. Allied Sci. 52, 153-158 (2010).

23. Biddie, S. C., Conway-Campbell, B. L. \& Lightman, S. L. Dynamic regulation of glucocorticoid signalling in health and disease. Rheumatology 51, 403-412 (2011).

24. Ramamoorthy, S. \& Cidlowski, J. A. Corticosteroids: mechanisms of action in health and disease. Rheum. Dis. Clin. 42, 15-31 (2016)

25. Dooley, D. P., Carpenter, J. L. \& Rademacher, S. Adjunctive corticosteroid therapy for tuberculosis: a critical reappraisal of the literature. Clin. Infect. Dis. 25, 872-887 (1997)

26. Horne, N. W. Prednisolone in treatment of pulmonary tuberculosis: a controlled trial. Br. Med. J. 2, 1751 (1960).

27. Lavers, K. W. \& Roberts, J. C. The use of prednisone in primary tuberculosis in children. Tubercle 40, 173-176 (1959).

28. Meintjes, G. et al. Randomized placebo-controlled trial of prednisone for paradoxical TB-associated immune reconstitution inflammatory syndrome. AIDS Lond. Engl. 24, 2381 (2010).

29. Kumarvelu, S., Prasad, K., Khosla, A., Behari, M. \& Ahuja, G. K. Randomized controlled trial of dexamethasone in tuberculous meningitis. Tuber. Lung Dis. J. Int. Union Tuberc. Lung Dis. 75, 203-207 (1994).

30. Mayosi, B. M. et al. Prednisolone and Mycobacterium indicus pranii in Tuberculous Pericarditis. N. Engl. J. Med. 371, 1121-1130 (2014).

31. Xie, S. et al. The efficacy and safety of adjunctive corticosteroids in the treatment of tuberculous pleurisy: a systematic review and meta-analysis. Oncotarget 8, 83315 (2017).

32. Critchley, J. A., Young, F., Orton, L. \& Garner, P. Corticosteroids for prevention of mortality in people with tuberculosis: a systematic review and meta-analysis. Lancet Infect. Dis. 13, 223-237 (2013).

33. Page, C. P. \& Spina, D. Phosphodiesterase inhibitors in the treatment of inflammatory diseases. Handb. Exp. Pharmacol. 391-414 (2011). https://doi.org/ 10.1007/978-3-642-17969-3_17 (2011).

34. Maiga, M. et al. Successful shortening of tuberculosis treatment using adjuvant host-directed therapy with FDA-approved phosphodiesterase inhibitors in the mouse model. PLOS ONE 7, e30749 (2012).

35. Maiga, M. C., Ahidjo, B. A., Maiga, M. \& Bishai, W. R. Roflumilast, a Type 4 phosphodiesterase inhibitor, shows promising adjunctive, host-directed therapeutic activity in a mouse model of tuberculosis. Antimicrob. Agents Chemother. 59, 7888-7890 (2015).

36. Subbian, $\mathrm{S}$. et al. Adjunctive phosphodiesterase-4 inhibitor therapy improves antibiotic response to pulmonary tuberculosis in a rabbit model. EBioMedicine $\mathbf{4}$, 104-114 (2016).

37. Subbian, S. et al. Phosphodiesterase-4 inhibition combined with isoniazid treatment of rabbits with pulmonary tuberculosis reduces macrophage activation and lung pathology. Am. J. Pathol. 179, 289-301 (2011).

38. Serafini, P. et al. Phosphodiesterase- 5 inhibition augments endogenous antitumor immunity by reducing myeloid-derived suppressor cell function. J. Exp. Med. 203, 2691-2702 (2006)

39. Cazzola, M. et al. Influence of $\mathrm{N}$-acetylcysteine on chronic bronchitis or COPD exacerbations: a meta-analysis. Eur. Respir. Rev. 24, 451-461 (2015).

40. Amaral, E. P. et al. $\mathrm{N}$-acetyl-cysteine exhibits potent anti-mycobacterial activity in addition to its known anti-oxidative functions. BMC Microbiol. 16, 251 (2016).

41. Teskey, G. et al. The synergistic effects of the glutathione precursor, NAC and first-line antibiotics in the granulomatous response against Mycobacterium tuberculosis. Front. Immunol. 9, 2069-2069 (2018).

42. Mahakalkar, S. M. et al. $\mathrm{N}$-acetylcysteine as an add-on to directly observed therapy short-I therapy in fresh pulmonary tuberculosis patients: a randomized, placebo-controlled, double-blinded study. Perspect. Clin. Res. 8, 132 (2017).

43. Cheng, S.-L. Protective effect of $\mathrm{N}$-acetylcysteine on antituberculosis drug induced hepatotoxicity. Eur. Respir. J. 48, PA2716 (2016).

44. Baniasadi, S. et al. Protective effect of N-acetylcysteine on antituberculosis druginduced hepatotoxicity. Eur. J. Gastroenterol. Hepatol. 22, 1235-1238 (2010).
45. Vilchèze, C. et al. Enhanced respiration prevents drug tolerance and drug resistance in Mycobacterium tuberculosis. Proc. Natl. Acad. Sci. USA 114 4495-4500 (2017)

46. Yogalingam, G. \& Pendergast, A. M. Abl kinases regulate autophagy by promoting the trafficking and function of lysosomal components. J. Biol. Chem. 283, 35941-35953 (2008).

47. Bruns, $H$. et al. Abelson tyrosine kinase controls phagosomal acidification required for killing of Mycobacterium tuberculosis in human macrophages. $J$. Immunol. 189, 4069-4078 (2012).

48. Napier, R. J. et al. Low doses of imatinib induce myelopoiesis and enhance host anti-microbial immunity. PLoS Pathog. 11, e1004770 (2015).

49. Napier, R. J. et al. Imatinib-sensitive tyrosine kinases regulate mycobacterial pathogenesis and represent therapeutic targets against tuberculosis. Cell Host Microbe 10, 475-485 (2011).

50. Steiger, J. et al. Imatinib triggers phagolysosome acidification and antimicrobial activity against Mycobacterium bovis bacille Calmette-Guérin in glucocorticoidtreated human macrophages. J. Immunol. 197, 222-232 (2016).

51. Sogi, K. M., Lien, K. A., Johnson, J. R., Krogan, N. J. \& Stanley, S. A. The tyrosine kinase inhibitor gefitinib restricts Mycobacterium tuberculosis growth through increased lysosomal biogenesis and modulation of cytokine signaling. ACS Infect. Dis. 3, 564-574 (2017).

52. Hussain, T. et al. Nilotinib: a tyrosine kinase inhibitor mediates resistance to intracellular Mycobacterium Via regulating autophagy. Cells 8, 506 (2019).

53. Zheng, C., Hu, M. \& Gao, F. Diabetes and pulmonary tuberculosis: a global overview with special focus on the situation in Asian countries with high TB-DM burden. Glob. Health Action 10, 1264702 (2017).

54. Stevenson, C. R. et al. Diabetes and tuberculosis: the impact of the diabetes epidemic on tuberculosis incidence. BMC Public Health 7, 234 (2007).

55. Hsieh, C.-H., He, C.-T., Lee, C.-H., Wu, L.-Y. \& Hung, Y.-J. Both slow-release and regular-form metformin improve glycemic control without altering plasma visfatin level in patients with type 2 diabetes mellitus. Metabolism 56, 1087-1092 (2007).

56. Marupuru, S. et al. Protective effect of metformin against tuberculosis infections in diabetic patients: an observational study of south Indian tertiary healthcare facility. Braz. J. Infect. Dis. Off. Publ. Braz. Soc. Infect. Dis. 21, 312-316 (2017).

57. Hirst, J. A., Farmer, A. J., Ali, R., Roberts, N. W. \& Stevens, R. J. Quantifying the effect of metformin treatment and dose on glycemic control. Diabetes Care 35, 446-454 (2012).

58. Lee, Y.-J. et al. The effect of metformin on culture conversion in tuberculosis patients with diabetes mellitus. Korean J. Intern. Med. 33, 933 (2018)

59. Martinez, N., Ketheesan, N., West, K., Vallerskog, T. \& Kornfeld, H. Impaired Recognition of Mycobacterium tuberculosis by Alveolar Macrophages From Diabetic Mice. J. Infect. Dis. 214, 1629-1637 (2016).

60. Martens, G. W. et al. Tuberculosis Susceptibility of Diabetic Mice. Am. J. Respir. Cell Mol. Biol. 37, 518-524 (2007).

61. Lopez-Lopez, N. et al. Type-2 diabetes alters the basal phenotype of human macrophages and diminishes their capacity to respond, internalise, and control Mycobacterium tuberculosis. Mem. Inst. Oswaldo Cruz 113, e170326-e170326 (2018).

62. Restrepo, B. I., Twahirwa, M., Rahbar, M. H. \& Schlesinger, L. S. Phagocytosis via complement or Fc-Gamma receptors is compromised in monocytes from type 2 diabetes patients with chronic hyperglycemia. PLoS ONE 9, e92977 (2014).

63. Zhou, G. et al. Role of AMP-activated protein kinase in mechanism of metformin action. J. Clin. Invest. 108, 1167-1174 (2001).

64. Kukidome, D. et al. Activation of AMP-activated protein kinase reduces hyperglycemia-induced mitochondrial reactive oxygen species production and promotes mitochondrial biogenesis in human umbilical vein endothelial cells. Diabetes 55, 120-127 (2006).

65. Singhal, A. et al. Metformin as adjunct antituberculosis therapy. Sci. Transl. Med. 6, 263ra159- (2014)

66. Vashisht, R. \& Brahmachari, S. K. Metformin as a potential combination therapy with existing front-line antibiotics for Tuberculosis. J. Transl. Med. 13, 83 (2015).

67. Degner, N. R., Wang, J.-Y., Golub, J. E. \& Karakousis, P. C. Metformin use reverses the increased mortality associated with diabetes mellitus during tuberculosis treatment. Clin. Infect. Dis. 66, 198-205 (2017).

68. Pan, S.-W. et al. The risk of TB in patients with type 2 diabetes initiating metformin vs. sulfonylurea treatment. Chest. https://doi.org/10.1016/j. chest.2017.11.040 (2017).

69. Lachmandas, E. et al. Metformin alters human host responses to Mycobacterium tuberculosis in healthy subjects. J. Infect. Dis. 220, 139-150 (2019).

70. Adak, T., Samadi, A., Ünal, A. Z. \& Sabuncuoğlu, S. A reappraisal on metformin. Regul. Toxicol. Pharmacol. 92, 324-332 (2018). 
71. Tukvadze, N. et al. High-dose vitamin D3 in adults with pulmonary tuberculosis: a double-blind randomized controlled trial. Am. J. Clin. Nutr. 102, 1059-1069 (2015).

72. Wejse, C. et al. Vitamin D as supplementary treatment for tuberculosis: a doubleblind, randomized, placebo-controlled trial. Am. J. Respir. Crit. Care Med. 179, 843-850 (2009).

73. Yuk, J.-M. et al. Vitamin D3 induces autophagy in human monocytes/macrophages via cathelicidin. Cell Host Microbe 6, 231-243 (2009).

74. Liu, P. T., Stenger, S., Tang, D. H. \& Modlin, R. L. Cutting edge: vitamin Dmediated human antimicrobial activity against Mycobacterium tuberculosis is dependent on the induction of cathelicidin. J. Immunol. 179, 2060-2063 (2007).

75. Lawson, L. et al. Randomized controlled trial of zinc and vitamin A as coadjuvants for the treatment of pulmonary tuberculosis. Trop. Med. Int. Health 15, 1481-1490 (2010).

76. Villamor, E. et al. A trial of the effect of micronutrient supplementation on treatment outcome, $T$ cell counts, morbidity, and mortality in adults with pulmonary tuberculosis. J. Infect. Dis. 197, 1499-1505 (2008).

77. Iannitti, T. \& Palmieri, B. Clinical and Experimental Applications of Sodium Phenylbutyrate. Drugs RD 11, 227-249 (2011).

78. Peña-Quintana, L., Llarena, M., Reyes-Suárez, D. \& Aldámiz-Echevarria, L. Profile of sodium phenylbutyrate granules for the treatment of urea-cycle disorders: patient perspectives. Patient Prefer. Adherence 11, 1489-1496 (2017).

79. Aibana, O. et al. Impact of vitamin A and carotenoids on the risk of tuberculosis progression. Clin. Infect. Dis. 65, 900-909 (2017).

80. Coussens, A. K. et al. High-dose vitamin D3 reduces deficiency caused by low UVB exposure and limits HIV-1 replication in urban Southern Africans. Proc. Natl. Acad. Sci. USA 112, 8052-8057 (2015).

81. Rekha, R. S. et al. Phenylbutyrate induces LL-37-dependent autophagy and intracellular killing of Mycobacterium tuberculosis in human macrophages. Autophagy 11, 1688-1699 (2015).

82. Mahoney, K. M., Freeman, G. J. \& McDermott, D. F. The next immune-checkpoint inhibitors: PD-1/PD-L1 blockade in melanoma. Clin. Ther. 37, 764-782 (2015).

83. Dyck, L. \& Mills, K. H. Immune checkpoints and their inhibition in cancer and infectious diseases. Eur. J. Immunol. 47, 765-779 (2017).

84. Jayaraman, P. et al. TIM3 mediates T cell exhaustion during Mycobacterium tuberculosis infection. PLoS Pathog. 12, e1005490 (2016).

85. Kirman, J. et al. CTLA-4 blockade enhances the immune response induced by mycobacterial infection but does not lead to increased protection. Infect. Immun. 67, 3786-3792 (1999).

86. Jurado, J. O. et al. Programmed death (PD)-1: PD-ligand 1/PD-ligand 2 pathway inhibits $T$ cell effector functions during human tuberculosis. J. Immunol. 181, 116-125 (2008).

87. Reljic, R., Paul, M. J. \& Arias, M. A. Cytokine therapy of tuberculosis at the crossroads. Expert Rev. Respir. Med. 3, 53-66 (2009).

88. Rivero-Lezcano, O. M. Cytokines as immunomodulators in tuberculosis therapy. Recent Patents Anti-Infect. Drug Disc. 3, 168-176 (2008).

89. Fujita, K., Terashima, T. \& Mio, T. Anti-PD1 antibody treatment and the development of acute pulmonary tuberculosis. J. Thorac. Oncol. 11, 2238-2240 (2016).

90. Benmerzoug, S. et al. GM-CSF targeted immunomodulation affects host response to M. tuberculosis infection. Sci. Rep. 8, 8652-8652 (2018).

91. Kumar, N. P., Moideen, K., Banurekha, V. V., Nair, D. \& Babu, S. Plasma proinflammatory cytokines are markers of disease severity and bacterial burden in pulmonary tuberculosis. Open Forum Infect. Dis. 6, 1-8 (2019).

92. Holland, S. M. et al. Treatment of refractory disseminated nontuberculous mycobacterial infection with interferon gamma: a preliminary report. $\mathrm{N}$. Engl. J. Med. 330, 1348-1355 (1994).

93. Dawson, R. et al. Immunomodulation with recombinant interferon-gamma1b in pulmonary tuberculosis. PLOS ONE 4, e6984 1-8 (2009).

94. Condos, R. \& Schluger, N. W. Cytokine-Based Approaches to the Treatment of Multidrug-Resistant Tuberculosis. BioDrugs 11, 165-173 (1999).

95. Johnson, B. J. et al. Clinical and immune responses of tuberculosis patients treated with low-dose IL-2 and multidrug therapy. Cytokines Mol. Ther. 1, 185-196 (1995)

96. Pedral-Sampaio, D. B. et al. Use of Rhu-GM-CSF in Pulmonary Tuberculosis Patients: Results of a Randomized Clinical Trial. Braz J Infect Dis. 7, 245-252 (2003).

97. Johnson, J. L. et al. Randomized trial of adjunctive interleukin-2 in adults with pulmonary tuberculosis. Am. J. Respir. Crit. Care Med. 168, 185-191 (2003).

98. Sakai, S. et al. CD4 T cell-derived IFN- $\gamma$ plays a minimal role in control of pulmonary Mycobacterium tuberculosis infection and must be actively repressed by PD-1 to prevent lethal disease. PLoS Pathog. 12, e1005667 (2016).

99. Roca, F. J. \& Ramakrishnan, L. TNF dually mediates resistance and susceptibility to mycobacteria through mitochondrial reactive oxygen species. Cell 153, 521-534 (2013)
100. Blackmore, T. K., Manning, L., Taylor, W. J. \& Wallis, R. S. Therapeutic use of infliximab in tuberculosis to control severe paradoxical reaction of the brain and lymph nodes. Clin. Infect. Dis. 47, e83-e85 (2008).

101. Wallis, R. S., van Vuuren, C. \& Potgieter, S. Adalimumab treatment of lifethreatening tuberculosis. Clin. Infect. Dis. Off. Publ. Infect. Dis. Soc. Am. 48, 1429-1432 (2009).

102. Wallis, R. S. Tumour necrosis factor antagonists: structure, function, and tuberculosis risks. Lancet Infect. Dis. 8, 601-611 (2008).

103. Zhang, Z. et al. Risk of tuberculosis in patients treated with TNF-a antagonists: a systematic review and meta-analysis of randomised controlled trials. BMJ Open 7, e012567 (2017)

104. Rivero-Lezcano, O. M. Cytokines as immunomodulators in tuberculosis therapy. Recent Patents Anti-Infect. Drug Disc. 3, 168-176 (2008)

105. Parihar, S. P. et al. Statin therapy reduces the mycobacterium tuberculosis burden in human macrophages and in mice by enhancing autophagy and phagosome maturation. J. Infect. Dis. 209, 754-763 (2014).

106. Skerry, C. et al. Simvastatin increases the in vivo activity of the first-line tuberculosis regimen. J. Antimicrob. Chemother. 69, 2453-2457 (2014).

107. Lobato, L. S. et al. Statins increase rifampin Mycobactericidal effect. Antimicrob. Agents Chemother. 58, 5766-5774 (2014).

108. Kyrklund, C. et al. Rifampin greatly reduces plasma simvastatin and simvastatin acid concentrations. Clin. Pharmacol. Ther. 68, 592-597 (2000).

109. Rao, M. et al. Improving treatment outcomes for MDR-TB-novel host-directed therapies and personalised medicine of the future. Int. J. Infect. Dis. 80, S62-S67 (2019).

110. Skrahin, A. et al. Autologous mesenchymal stromal cell infusion as adjunct treatment in patients with multidrug and extensively drug-resistant tuberculosis: an open-label phase 1 safety trial. Lancet Respir. Med. 2, 108-122 (2014).

111. Skrahin, A. E. et al. Potential role of autologous mesenchymal stromal cells in the treatment of multidrug and extensively drug-resistant tuberculosis. Eur. Respir. J. 48, PA1919 (2016)

112. Mizukami, A. \& Swiech, K. Mesenchymal stromal cells: from discovery to manufacturing and commercialization. Stem Cells Int. 2018 (2018).

113. Iannaccone, M., Dorhoi, A. \& Kaufmann, S. H. E. Host-directed therapy of tuberculosis: what is in it for microRNA? Expert Opin. Ther. Targets 18, 491-494 (2014).

114. Desikan, P. \& Rangnekar, A. Host-targeted therapy for tuberculosis: time to revisit the concept. Indian J. Med. Res. 147, 233 (2018).

115. Paik, S., Kim, J. K., Chung, C. \& Jo, E.-K. Autophagy: a new strategy for hostdirected therapy of tuberculosis. Virulence 10, 448-459 (2019).

116. Stanley, S. A. et al. Identification of host-targeted small molecules that restrict intracellular Mycobacterium tuberculosis growth. PLoS Pathog. 10, e1003946 (2014).

117. Zhang, Q. et al. Antimycobacterial and anti-inflammatory mechanisms of baicalin via induced autophagy in macrophages infected with mycobacterium tuberculosis. Front. Microbiol. 8, 2142 (2017).

118. Juarez, E. et al. Loperamide restricts intracellular growth of Mycobacterium tuberculosis in lung macrophages. Am. J. Respir. Cell Mol. Biol. 55, 837-847 (2016).

119. Kim, J.-J. et al. Host cell autophagy activated by antibiotics is required for their effective antimycobacterial drug action. Cell Host Microbe 11, 457-468 (2012).

120. Choi, S. W. et al. Ambroxol induces autophagy and potentiates rifampin antimycobacterial activity. Antimicrob. Agents Chemother. 62, e01019-18 (2018).

121. Walker, N. F. et al. Doxycycline and HIV infection suppress tuberculosis-induced matrix metalloproteinases. Am. J. Respir. Crit. Care Med. 185, 989-997 (2012).

122. Ong, C. W. et al. Neutrophil-derived MMP-8 drives AMPK-dependent matrix destruction in human pulmonary tuberculosis. PLoS Pathog. 11, e1004917 (2015).

123. Nemes, E. et al. Prevention of M. tuberculosis infection with $\mathrm{H} 4$ : IC31 vaccine or BCG revaccination. N. Engl. J. Med. 379, 138-149 (2018).

124. Kleinnijenhuis, J. et al. Bacille Calmette-Guerin induces NOD2-dependent nonspecific protection from reinfection via epigenetic reprogramming of monocytes. Proc. Natl. Acad. Sci. USA 109, 17537-17542 (2012).

125. Joosten, S. A. et al. Mycobacterial growth inhibition is associated with trained innate immunity. J. Clin. Invest. 128 (2018).

126. Goodridge, H. S. et al. Harnessing the beneficial heterologous effects of vaccination. Nat. Rev. Immunol. 16, 392 (2016).

127. Benn, C. S., Netea, M. G., Selin, L. K. \& Aaby, P. A small jab-a big effect: nonspecific immunomodulation by vaccines. Trends Immunol. 34, 431-439 (2013).

128. Tanner, R., Villarreal-Ramos, B., Vordermeier, M. \& McShane, H. The humoral immune response to BCG vaccination. Front. Immunol. 10, 1317 (2019). 
129. Koeken, V. A., Verrall, A. J., Netea, M. G., Hill, P. C. \& van Crevel, R. Trained innate immunity and resistance to Mycobacterium tuberculosis infection. Clin. Microbiol. Infect. In press (2019).

130. Schaible, U. E., Linnemann, L., Redinger, N., Patin, E. C. \& Dallenga, T. Strategies to improve vaccine efficacy against Tuberculosis by Targeting innate immunity. Front. Immunol. 8, 1755 (2017).

131. Vilaplana, C. et al. Ibuprofen therapy resulted in significantly decreased tissue bacillary loads and increased survival in a new murine experimental model of active tuberculosis. J. Infect. Dis. 208, 199-202 (2013).

132. Eisen, D. P., McBryde, E. S. \& Walduck, A. Low-dose aspirin and ibuprofen's sterilizing effects on mycobacterium tuberculosis suggest safe new adjuvant therapies for tuberculosis. J. Infect. Dis. 208, 1925-1927 (2013).

133. Byrne, S. T., Denkin, S. M. \& Zhang, Y. Aspirin and ibuprofen enhance pyrazinamide treatment of murine tuberculosis. J. Antimicrob. Chemother. 59, 313-316 (2006).

134. Misra, U. K., Kalita, J. \& Nair, P. P. Role of aspirin in tuberculous meningitis: a randomized open label placebo controlled trial. J. Neurol. Sci. 293, 12-17 (2010).

135. Schoeman, J. F., Janse van Rensburg, A., Laubscher, J. A. \& Springer, P. The role of aspirin in childhood tuberculous meningitis. J. Child Neurol. 26, 956-962 (2011).

136. Peres-Buzalaf, C. et al. Control of experimental pulmonary tuberculosis depends more on immunostimulatory leukotrienes than on the absence of immunosuppressive prostaglandins. Prostaglandins Leukot. Essent. Fatty Acids 85, 75-81 (2011).

137. Kalle, A. M. \& Rizvi, A. Inhibition of bacterial multidrug resistance by celecoxib, a cyclooxygenase-2 inhibitor. Antimicrob. Agents Chemother. 55, 439-442 (2011).

138. Salunke, S. B. et al. Design and synthesis of novel anti-tuberculosis agents from the celecoxib pharmacophore. Bioorg. Med. Chem. 23, 1935-1943 (2015).

139. Semba, R. D. et al. Micronutrient supplements and mortality of HIV-infected adults with pulmonary TB: a controlled clinical trial. Int. J. Tuberc. Lung Dis. 11, 854-859 (2007).

140. Martineau, A. R. et al. High-dose vitamin D3 during intensive-phase antimicrobial treatment of pulmonary tuberculosis: a double-blind randomised controlled trial. The Lancet 377, 242-250 (2011).

141. Daley, P. et al. Adjunctive vitamin D for treatment of active tuberculosis in India: a randomised, double-blind, placebo-controlled trial. Lancet Infect. Dis. 15, 528-534 (2015).

142. Kamen, D. L. \& Tangpricha, V. Vitamin D and molecular actions on the immune system: modulation of innate and autoimmunity. J. Mol. Med. 88, 441-450 (2010).

143. Grange, J. M., Davies, P. D. O., Brown, R. C., Woodhead, J. S. \& Kardjito, T. A study of vitamin $\mathrm{D}$ levels in Indonesian patients with untreated pulmonary tuberculosis. Tubercle 66, 187-191 (1985).

144. Salahuddin, N. et al. Vitamin D accelerates clinical recovery from tuberculosis: results of the SUCCINCT Study [Supplementary Cholecalciferol in recovery from tuberculosis]. A randomized, placebo-controlled, clinical trial of vitamin D supplementation in patients with pulmonary tuberculosis'. BMC Infect. Dis. 13, 22 (2013).

145. Ralph, A. P. et al. L-arginine and vitamin D adjunctive therapies in pulmonary tuberculosis: a randomised, double-blind, placebo-controlled trial. PloS One 8 e70032 (2013).

146. Schön, T. et al. Arginine as an adjuvant to chemotherapy improves clinical outcome in active tuberculosis. Eur. Respir. J. 21, 483-488 (2003).

147. Mily, A. et al. Significant effects of oral phenylbutyrate and vitamin D3 adjunctive therapy in pulmonary tuberculosis: a randomized controlled trial. PLOS ONE 10, e0138340 (2015).

148. Coussens, A. K., Wilkinson, R. J. \& Martineau, A. R. Phenylbutyrate is bacteriostatic against Mycobacterium tuberculosis and regulates the macrophage response to infection, synergistically with 25 -hydroxy-vitamin $D_{3}$. PLoS Pathog. 11, e1005007 (2015)

149. Armijos, R. X., Weigel, M. M., Chacon, R., Flores, L. \& Campos, A. Adjunctive micronutrient supplementation for pulmonary tuberculosis. Salud Pública México 52, 185-189 (2010).

150. Range, N. et al. The effect of multi-vitamin/mineral supplementation on mortality during treatment of pulmonary tuberculosis: a randomised two-by-two factorial trial in Mwanza, Tanzania. Br. J. Nutr. 95, 762-770 (2006).

151. Overbeck, S., Rink, L. \& Haase, H. Modulating the immune response by oral zinc supplementation: a single approach for multiple diseases. Arch. Immunol. Ther. Exp. (Warsz.) 56, 15-30 (2008).

152. Arjanova, O. V. et al. Impact of adjunct immunotherapy with multi-herbal supplement Dzherelo (Immunoxel) on treatment outcomes in end-stage TB/HIV patients. J. Antivir. Antiretrovir. 1, 86-88 (2009).

153. Zaiteva, S. I. et al. Efficacy and safety of phytoconcentrate Dzherelo (Immunoxel) in treatment of patients with multi-drug resistant TB (MDR-TB) in comparison to standartd chemotherapy. Res. J. Med. Sci. 3, 36-41 (2009).
154. Nikolaeva, L. G. et al. Cytokine profiles of HIV patients with pulmonary tuberculosis resulting from adjunct immunotherapy with herbal phytoconcentrates Dzherelo and Anemin. Cytokine 44, 392-396 (2008).

155. Efremenko, Y. V. et al. Clinical validation of sublingual formulations of Immunoxel (Dzherelo) as an adjuvant immunotherapy in treatment of TB patients. Immunotherapy 4, 273-282 (2012).

156. Hakim, J. G. et al. Double blind randomised placebo controlled trial of adjunctive prednisolone in the treatment of effusive tuberculous pericarditis in HIV seropositive patients. Heart 84, 183-188 (2000).

157. Elliott, A. et al. Use of Prednisolone in the Treatment of HIV-positive Tuberculosis Patients. QJM Int. J. Med. 85, 855-860 (1992).

158. Bilaçeroğlu, S., Perim, K., Büyükşirin, M. \& Celikten, E. Prednisolone: a beneficial and safe adjunct to antituberculosis treatment? A randomized controlled trial. Int. J. Tuberc. Lung Dis. 3, 47-54 (1999).

159. Bell, W. J., Brown, P. P. \& Horn, D. W. Prednisolone in the treatment of acute extensive pulmonary tuberculosis in West Africans. Tubercle 41, 341-351 (1960).

160. Rock, R. B. et al. Mycobacterium tuberculosis-induced cytokine and chemokine expression by human microglia and astrocytes: effects of dexamethasone. J. Infect. Dis. 192, 2054-2058 (2005).

161. Girgis, N. I., Farid, Z., Kilpatrick, M. E., Sultan, Y. \& Mikhail, I. A. Dexamethasone adjunctive treatment for tuberculous meningitis. Pediatr. Infect. Dis. J. 10, 179-183 (1991).

162. Kesavan, A. K. et al. Effects of dexamethasone and transient malnutrition on rabbits infected with aerosolized Mycobacterium tuberculosis CDC1551. Infect. Immun. 73, 7056-7060 (2005).

163. Green, J. A. et al. Dexamethasone, cerebrospinal fluid matrix metalloproteinase concentrations and clinical outcomes in tuberculous meningitis. PLOS ONE 4, e7277 (2009).

164. Török, M. E. et al. Dexamethasone and long-term outcome of tuberculous meningitis in Vietnamese adults and adolescents. PLOS ONE 6, e27821 (2011).

165. Donovan, J. et al. Adjunctive dexamethasone for the treatment of HIV-infected adults with tuberculous meningitis (ACT HIV): study protocol for a randomised controlled trial. Wellcome Open Res. 3, 31 (2018).

166. Thwaites, G. E. et al. Dexamethasone for the treatment of tuberculous meningitis in adolescents and adults. N. Engl. J. Med. 351, 1741-1751 (2004).

167. Banerjee, D. \& Bhattacharyya, R. Statin therapy may prevent development of tuberculosis in diabetic state. Med. Hypotheses 83, 88-91 (2014).

168. Eiter, L. C. et al. Gold (I) analogues of a platinum-acridine antitumor agent are only moderately cytotoxic but show potent activity against Mycobacterium tuberculosis. J. Med. Chem. 52, 6519-6522 (2009).

169. Harbut, M. B. et al. Auranofin exerts broad-spectrum bactericidal activities by targeting thiol-redox homeostasis. Proc. Natl. Acad. Sci. USA 112, 4453-4458 (2015).

170. Subbian, S. et al. Pharmacologic inhibition of host phosphodiesterase-4 improves isoniazid-mediated clearance of Mycobacterium tuberculosis. Front. Immunol. 7, 238 (2016).

171. Subbian, S. et al. Phosphodiesterase-4 inhibition alters gene expression and improves isoniazid-mediated clearance of Mycobacterium tuberculosis in rabbit lungs. PLoS Pathog. 7, e1002262 (2011).

172. Koo, M.-S. et al. Phosphodiesterase 4 inhibition reduces innate immunity and improves isoniazid clearance of Mycobacterium tuberculosis in the lungs of infected mice. PLoS ONE 6, e17091 (2011).

173. Attri, S. et al. Isoniazid- and rifampicin-induced oxidative hepatic injuryprotection by $\mathrm{N}$-acetylcysteine. Hum. Exp. Toxicol. 19, 517-522 (2000).

174. Condos, R., Rom, W. N. \& Schluger, N. W. Treatment of multidrug-resistant pulmonary tuberculosis with interferon-gamma via aerosol. Lancet Lond. Engl. 349, 1513-1515 (1997).

175. Giosuè, S. et al. Aerosolized interferon-alpha treatment in patients with multi-drug-resistant pulmonary tuberculosis. Eur. Cytokine Netw. 11, 99-104 (2000).

176. Suárez-Méndez, R. et al. Adjuvant interferon gamma in patients with drugresistant pulmonary tuberculosis: a pilot study. BMC Infect. Dis. 4, 44 (2004).

177. Grahmann, P. R. \& Braun, R. K. A new protocol for multiple inhalation of IFNsuccessfully treats MDR-TB: a case study. Int. J. Tuberc. Lung Dis. 12, 636-644 (2008).

178. Wallis, R. S. et al. A study of the safety, immunology. virology, and microbiology of adjunctive etanercept in HIV-1-associated tuberculosis. AIDS Lond. Engl. 18, 257-264 (2004).

179. Mayanja-Kizza, H. et al. Immunoadjuvant prednisolone therapy for HIVassociated tuberculosis: a phase 2 clinical trial in Uganda. J. Infect. Dis. 191, 856-865 (2005).

180. Allen, S. S., Cassone, L., Lasco, T. M. \& McMurray, D. N. Effect of neutralizing transforming growth factor $\beta 1$ on the immune response against Mycobacterium tuberculosis in guinea pigs. Infect. Immun. 72, 1358-1363 (2004). 
181. Datta, M. et al. Anti-vascular endothelial growth factor treatment normalizes tuberculosis granuloma vasculature and improves small molecule delivery. Proc. Natl. Acad. Sci. USA 112, 1827-1832 (2015).

182. Oehlers, S. H. et al. Interception of host angiogenic signalling limits mycobacterial growth. Nature 517, 612-615 (2015).

183. Johnson, B. J. et al. rhulL-2 adjunctive therapy in multidrug resistant tuberculosis: a comparison of two treatment regimens and placebo. Tuber. Lung Dis. 78, 195-203 (1997).

184. Buccheri, S. et al. IL-4 depletion enhances host resistance and passive IgA protection against tuberculosis infection in BALB/c mice. Eur. J. Immunol. 37, 729-737 (2007).

185. Roy, E., Brennan, J., Jolles, S. \& Lowrie, D. B. Beneficial effect of anti-interleukin-4 antibody when administered in a murine model of tuberculosis infection. Tuberc. Edinb. Scotl. 88, 197-202 (2008).

186. Singh, V. et al. Co-administration of IL-1+IL-6+TNF-a with Mycobacterium tuberculosis infected macrophages vaccine induces better protective $\mathrm{T}$ cell memory than BCG. PLoS ONE 6, e16097 (2011).

187. Maeurer, M. J. et al. Interleukin-7 or Interleukin-15 Enhances Survival of Mycobacterium tuberculosis-Infected Mice. Infect. Immun. 68, 2962-2970 (2000).

188. Silva, R. A., Pais, T. F. \& Appelberg, R. Blocking the receptor for IL-10 improves antimycobacterial chemotherapy and vaccination. J. Immunol. 167, 1535-1541 (2001).

189. Flynn, J. L. et al. IL-12 increases resistance of BALB/c mice to Mycobacterium tuberculosis infection. J. Immunol. 155, 2515-2524 (1995).

190. Greinert, U., Ernst, M., Schlaak, M. \& Entzian, P. Interleukin-12 as successful adjuvant in tuberculosis treatment. Eur. Respir. J. 17, 1049-1051 (2001).

191. Umemura, M. et al. Interleukin-15 as an immune adjuvant to increase the efficacy of Mycobacterium bovis bacillus Calmette-Guérin vaccination. Infect. Immun. 71, 6045-6048 (2003).

192. Happel, K. I. et al. Pulmonary interleukin-23 gene delivery increases local T-cell immunity and controls growth of Mycobacterium tuberculosis in the lungs. Infect. Immun. 73, 5782-5788 (2005).
193. Ma, Y. et al. Interleukin 24 as a novel potential cytokine immunotherapy for the treatment of Mycobacterium tuberculosis infection. Microbes Infect. 13, 1099-1110 (2011).

194. Liu, H. et al. IL-37 Confers protection against Mycobacterial infection involving suppressing inflammation and modulating T cell activation. PLOS ONE 12, e0169922 (2017)

195. Shen, $H$. et al. The beneficial effects of adjunctive recombinant human interleukin-2 for multidrug resistant tuberculosis. Arch. Med. Sci. AMS 11, 584-590 (2015).

196. Tan, Q. et al. Clinical and immunological effects of rhlL-2 therapy in eastern Chinese patients with multidrug-resistant tuberculosis. Sci. Rep. 7, 17854 (2017).

197. Yoshino, S., Murata, Y. \& Ohsawa, M. Successful induction of adjuvant arthritis in mice by treatment with a monoclonal antibody against IL-4. J. Immunol. 161, 6904-6908 (1998).

Open Access This article is licensed under a Creative Commons Attribution 4.0 International License, which permits use, sharing, adaptation, distribution and reproduction in any medium or format, as long as you give appropriate credit to the original author(s) and the source, provide a link to the Creative Commons license, and indicate if changes were made. The images or other third party material in this article are included in the article's Creative Commons license, unless indicated otherwise in a credit line to the material. If material is not included in the article's Creative Commons license and your intended use is not permitted by statutory regulation or exceeds the permitted use, you will need to obtain permission directly from the copyright holder. To view a copy of this license, visit http://creativecommons. org/licenses/by/4.0/.

(c) The Author(s) 2019 